\title{
Thesen zu „Ethischer Politikberatung“
}

\author{
Katarina Weilert
}

\section{Kernaussagen}

Ethische Politikberatung umfasst sowohl eine wissensbasierte, als auch eine bewertende Ebene. Der Staat ist auf die Beachtung von Werten angewiesen, eine Negierung würde zu Intoleranz und Unfreiheit führen. Die letzte Entscheidung darüber, welche Wertmaßstäbe anzulegen sind, ist jedoch nicht Sache der Politikberatung, sondern der demokratisch legitimierten Parlamente.

\section{Einleitung}

Was ist eigentlich „ethische Politikberatung“ und wie funktioniert sie? Obwohl es kaum einen Mangel an wissenschaftlichen Abhandlungen zur „Politikberatung“ gibt, hat die Frage nach einer ethischen Politikberatung auf der Reflexionsebene bisher kaum Beachtung gefunden. Um diesem Defizit Abhilfe zu schaffen, müssen interdisziplinäre Zugänge zum Thema gesucht werden. ${ }^{1}$

„Ethische“ Politikberatung kann auf verschiedene Weise verstanden werden: Zum einen als eine Beratung in „ethisch relevanten Politikbereichen“, als Modus der Politikberatung und als Kennzeichnung von ethischen Politikberatungsakteuren. Im Fokus stehen hier daher sowohl die ethische Fragen in besonderer Weise tangierenden Politikberbereiche wie die Bioethik, Gesundheitspolitik und Umweltethik, als auch die verschiedenen Akteure, Instrumente und Formen ethischer Politikberatung. Im Folgenden werden auf dieser Grundlage acht Thesen zur „ethischen Politikberatung“ präsentiert.

\section{Acht Thesen über das Zusammenwirken von Werten und Wissen}

\subsection{Politikberatung kann Werte vermitteln}

Politikberatung kann das Werteberücksichtigungspotential in der Politik bei wissenschafts- und technikrelevanten Kontroversen steigern. Diese Meinung vertritt Stephan Bröchler (Gießen) und befindet, dass die Politik unter einem permanenten Entscheidungszwang stehe, Entscheidungen sowohl zu treffen als auch durchzusetzen, obwohl es viele Unsicherheiten gebe. Die Erwartungen der Politik an die Berater und insbesondere die wissenschaftliche Politikberatung sind sehr vielfältig: Politikberatung solle Probleme rechtzeitig erkennen, Prognosen liefern und bereits getroffene Entscheidungen wissenschaftlich autorisieren. Politikberatung müsse mitunter aber auch als „Feigenblatt“ dienen, um Entscheidungen als „Sachzwang“ darzustellen und müsse überdies die Politik evaluieren und kontrollieren.
Ziel der modernen ethischen Politikberatung sei es, (wissenschaftliche) Informationen für eine verantwortliche, allgemein verbindliche Entscheidung bei öffentlichen Konflikten anzubieten. Die Beratergruppen sind dabei sehr vielfältig und sehr unterschiedlich in ihrer Art. Sie reichen von institutionalisierten Beiräten und Kommissionen bis hin zu „Laien“-Experten. Auch die Formen der Politikberatung gestalten sich verschieden. Informelle Gespräche stehen neben Ad-hocAusschüssen oder einer offiziellen institutionalisierten Beratung. $\mathrm{Zu}$ unterscheiden ist zwischen Interessen-, Wissensund Wertkonflikten.

Bei Wertkonflikten, so Bröchler, gebe es einen anerkannten Dissens über moralische Richtigkeitsbehauptungen. Gerade in Wertkonflikten sieht er auch Raum für Laienberatung, die bislang noch zu wenig erschlossen ist. Mit ihr wird die Öffentlichkeit aus ihrer Publikumsrolle herausgeholt. Experten dürfen dabei jedoch den legitimierten Institutionen nicht unangemessen vorgreifen. Ein Monitoring, das diese Punkte im Blick hat, sei ein notwendiger Kontrollmechanismus.

\subsection{Ethische Konflikte sind nicht nur Wissensprobleme, sondern vor allem Wertungsprobleme}

Gesellschaftspolitisch derzeit besonders relevant und bekannt sind Ethikräte und Ethikkommissionen. Hier treten zum einen zahlreiche Ad-hoc-Kommissionen oder ständige Kommissionen im Blick auf bestimmte ethische Entscheidungsszenarien auf, wie etwa aktuell die Ethikkommission für eine sichere Energieversorgung. Daneben steht der Deutsche Ethikrat, welcher, wie Hildegund Holzheid (Mitglied des Deutschen Ethikrates) erläuterte, nicht auf bestimmte ethische Bereiche beschränkt ist, wenngleich die ethischen Fragen in den Lebenswissenschaften bislang einen absolut dominanten Raum eingenommen haben. Beim Deutschen Ethikrat stehe nicht der Rationalitätsgewinn durch die Wissenschaft im Vordergrund, sondern die Repräsentanz verschiedener gesellschaftlicher Gruppierungen. Dies zeigt, dass ethische Konflikte nicht nur Wissensprobleme, sondern oft vor allem Wertungsprobleme sind.

Eine verantwortbare Entscheidung setzt eine umfassende Information bei den verschiedenen Disziplinen voraus. Daher ist das 26-köpfige Gremium interdisziplinär aufgestellt. Verantwortbarkeit verlange nach Vorurteilsfreiheit und Diskurs,

\footnotetext{
1 Diesem Beitrag liegen die Ergebnisse einer internationalen Expertentagung der FEST (Forschungsstätte der Evangelischen Studiengemeinschaft) und der HSS (Hanns-Seidel-Stiftung) zu Grunde, die im Dezember 2010 in Heidelberg stattfand. Die Thesen geben die Kernaussagen der genannten Referentinnen und Referenten wieder.
} 
wobei versucht werden müsse, die verschiedenen Sichtweisen zu verstehen. Weitere wesentliche Merkmale des Deutschen Ethikrates sind seine Unabhängigkeit, die Distanz zur Politik sowie die Tatsache, dass es keine inhaltlichen ethischen Vorgaben gibt. Besonders hervorzuheben ist laut Holzheid die Bedeutung von dissenting votes. Schließlich sei es gerade nicht die Aufgabe des Ethikrates, einen Konsens zu formulieren. Vielmehr sollen Lösungsmöglichkeiten und Handlungsoptionen erarbeitet werden. Wichtig ist, dass am Ende die Parlamente als demokratisch legitimierte Organe die Entscheidung treffen, womit zugleich der lediglich politikberatende Charakter des Rates verdeutlicht wird.

\subsection{Ethikkommissionen moderieren den Dissens}

Im Hinblick auf die Bioethikkommission beim österreichischen Bundeskanzleramt argumentiert Stefan Zotti (Brüssel), dass diese keine Ethik in die Politik bringe, sondern für ein grundsätzliches Verständnis von Ethik und Ethischem sorge. Es gehe ihr darum, Prozesse zu rationalisieren und eine seriöse politische Beobachtung überhaupt möglich zu machen. Die Kommission verfasst Empfehlungen und Stellungnahmen und ist klar auf den Bundeskanzler und die Regierung hin ausgerichtet. Zotti spricht sich dafür aus, dass die Kommission mehr ein Expertengremium sein sollte als ein „Stakeholder-Gremium“, während Holzheid für den Deutschen Ethikrat vor allem die Bedeutung verschiedener gesellschaftlicher Gruppierungen im Hinblick auf „wertende Fragestellungen “ über das bloße Expertenwissen hinaus betont. Unterschiede zeigen sich auch in der Wahrnehmung der Ratschläge: Während die unmittelbare politische Relevanz der Stellungnahmen der österreichischen Bioethikkommission eher gering ist, reagiert der deutsche Gesetzgeber positiver auf die Berichte des Deutschen Ethikrates, wenngleich er sich auch nicht notwendigerweise dem Namen nach auf den Ethikrat bezieht. Übereinstimmend stellen Holzheid und Zotti fest, dass ein solches Gremium den Dissens moderiere, Begründungen liefere und teils pragmatische Handlungsoptionen eröffne. Während in Deutschland die Legitimation des Ethikrates durch die Qualität des Verfahrens erfolge, müsse in Österreich die Politik mit den Experten übereinstimmen, um glaubwürdig zu sein. Ein Defizit ist laut Zotti, dass bei der österreichischen Ethikkommission die Positionen oft aufgrund der Besetzung vorhersehbar sind.

\subsection{Direkte Demokratie stärkt zivilgesellschaftlich organisierte Politikberatung}

Einen besonderen Charakter hat ethische Wirtschafts- und Politikberatung in einem direktdemokratischen politischen System. Stephan Wirz (Zürich) zufolge ist eine zivilgesellschaftlich organisierte Politikberatung in der Schweiz stark verankert. Verfahrensrechtlich ist dies insbesondere über die sog. Vernehmlassungen, die besonders bei Verfassungs- und Gesetzesänderungen stattfinden, abgesichert sowie über außerparlamentarische Kommissionen. Bei der Vernehmlas- sung können sich Interessengruppen zu Wort melden, sie ist somit als Form der zivilgesellschaftlich organisierten Politikberatung zu bezeichnen.

Das Selbstverständnis der Bürger zeichne sich in der Schweiz dadurch aus, dass die "Gleichheit“ (geschichtlich gegen die Aristokratie und Monarchie „ertrotzt“) besonders hoch angesiedelt ist. Daher fühlten sich die Bürger kompetent, in politischen Dingen zu urteilen und mitzuentscheiden. Die Verwobenheit von Bürgern und politischer Ebene erfolge nicht nur durch direktdemokratische Elemente, sondern auch durch die Wahrnehmung staatlicher Tätigkeiten durch gleichzeitig im zivilen Bereich Berufstätige. Ethische Politikberatung findet somit durch die Bürger statt und ist nicht vorrangig in die Hand von Experten gelegt.

\subsection{Der Staat ist auf Werte angewiesen}

Während in der Schweiz also die Ethikberatung vielfach „von unten“, vom Volk selbst aus, kommt, sind es in Deutschland mehr die institutionalisierten Gremien, die in der ethischen Politikberatung Einfluss haben. Wie sich zeigt, hat die ethische Politikberatung zwei Facetten: Zum einen kann eine verantwortbare Ethik nur auf der Grundlage wissenschaftlicher Informiertheit basieren, zum anderen aber gibt es einen unterschiedlichen Umgang mit wissenschaftlichen Erkenntnissen entsprechend der persönlichen ethischen Überzeugung. Vor diesem Hintergrund fragt Elisabeth Gräb-Schmidt (Tübingen), wessen Werte in einer pluralistischen Gesellschaft zählen, wenn es um wertgebundene Entscheidungen im modernen Verfassungsstaat geht.

Ausgangspunkt der Überlegungen ist die Prämisse, dass der Staat zwar weltanschaulich neutral, aber gleichwohl auf Werte angewiesen sei. Wertgebundenheit sei somit zentral und einem beliebigen Relativismus vorzuziehen. Nicht zuletzt bestehe die Gefahr der totalitären Ausschaltung von Werten, wenn sich der Staat „wertneutral“ verhält. Dies sei laut Gräb-Schmidt das Gegenteil von Toleranz, das Bekenntnis zum freiheitlichen Verfassungsstaat sei nicht disponibel.

Die Wertgebundenheit des Verfassungsstaates liege dabei in seinem Bekenntnis zur Menschenwürde und Freiheit. Werte sind nach Gräb-Schmidt jene Vorstellungen, welche in einer Gesellschaft allgemein als wünschenswert anerkannt sind und den Menschen Orientierung verleihen. Der Freiheit kommt hier eine Schlüsselposition zu. Entscheidend für die Geltungsfrage sei, wie sich ein Wert zur Freiheit verhält, wobei Werte nicht mit Traditionen zu verwechseln sind, auch wenn sich Werte in einem geschichtlichen und kulturellen Kontext entwickeln. Die Geltung von Werten finde dort ihre Grenze, wo Freiheits- und Würdeorientierung aufgehoben werden.

\subsection{Philosophie ist eine Herausforderung für die Politik}

Aus philosophischer Perspektive besteht ein „eigentümliches Verhältnis“ von Philosophie und Politik im Kontext ethischer Politikberatung. Das Verhältnis von Ethik und Politik ist 
schwierig, weil Ethik theoretisch ausgerichtet ist, Politik jedoch pragmatisch, so Marco Neher (Berlin). Die Sizilienreisen Platons zu Herrscher Dionysios II. von Syrakus stellten einen Versuch aktiver Politikberatung durch Platon dar, der jedoch scheiterte. Platon trat mit Forderungen an die Politik heran, die für die Politik nicht erfüllbar waren, da der Tyrann durch sie seine aktuelle gesellschaftliche Funktion verloren hätte.

Platon vergleicht die politische Beratertätigkeit mit der eines Arztes: Der Berater müsse wie der Arzt das Gute vor Augen haben. So wie für den Arzt die Gesundheit des ganzen Menschen im Vordergrund stehe, müsse der Berater ein Konzept des „guten Lebens“ anstreben. Nach Neher darf sich die Philosophie/Ethik nicht nur in der Ausbuchstabierung des Common Sense erschöpfen. Da die Philosophie keine Grenzen, zumindest keine politisch-gesellschaftlichen, anerkennen könne, sei sie immer auch eine Gefahr für die Politik. Das Verhältnis von Philosophie/Ethik zur Politik, so Neher, spiele schließlich auch jenseits von Einzelentscheidungen eine wichtige Rolle.

\subsection{Politikberater können Vermittler unterschiedlicher Ansprüche sein}

Das konkrete Feld der Umweltethik ist nützlich, um die Probleme der Realisierung ethischer Ansprüche in der Politik zu illustrieren. Ralf Tils (Lüneburg) plädiert dafür, dass die Politikberatung die einzelnen formulierten Ansprüche in eine positive Verbindung setzen müsse. Die verschiedenen Akteure umweltpolitischer Beratung reichen hinsichtlich ihres Institutionalisierungsgrades von gering („informelle Gespräche“) bis hoch (Kommissionen, Ressortforschung, etc.). Primäre Adressaten sind Regierung, Verwaltung und das Parlament.

In der umweltpolitischen Beratung gebe es die Besonderheit, dass aufgrund der sehr komplexen Fälle häufig ein Wissensproblem auftritt, das von unsicherem Wissen bis hin zu Nicht-Wissen reicht. In der Umweltpolitik würden, wie auch in anderen Politikbereichen, auf den politischen Entscheider eine Fülle an verschiedenen, meist separat vorgetragenen Ansprüchen zukommen. So werden ethische, fachliche, rechtliche, administrative und politische Ansprüche formuliert. Alle diese Ansprüche haben unterschiedliche primäre Interessen, ein guter Politikberater müsse demnach ein Mittler zwischen diesen verschiedenen Ansprüchen sein. Damit schlägt Tils einen neuen Typus des Politikberaters vor, der gleichsam nicht als Anwalt in eigener (fachlicher) Sache auftritt, sondern zwischen die verschiedenen Anspruchsteller und die Politik geschaltet ist.

\subsection{Bürgerbeteiligung steigert die Qualität der ethischen Politikberatung}

Silke Schicktanz (Göttingen) stellt die Frage, wie Politikberatung im Spannungsfeld zwischen Expertenwissen und Patienteninteressen aussieht bzw. aussehen müsste und widmet sich dabei dem Bereichsfeld der Bioethik und Gesundheitspo- litik. In Deutschland sind Experten für die ethische Politikberatung dominant vertreten, Patienten- und Behindertenverbände hingegen sind meist exkludiert und gerade Patienten werden nur selten als Kollektiv oder Gruppe angesehen. Schicktanz plädiert für eine größere Beteiligung von Bürgern im Rahmen der ethischen Politikberatung, da dadurch deren Qualität gesteigert werden könne. Beteiligung könne zu einer größeren Akzeptanz führen, da Grenzen individueller Rechte und Wertkonflikte besser vermittelt werden.

Auch die Kongruenz von Entscheidung und Betroffenen sei in diesem Zusammenhang positiv zu bewerten: Auf diese Weise finde eine Kopplung von Verantwortung und Entscheidung statt, zudem werden neue Werte- und Wissensbereiche entdeckt. Dem Einwand, Laien seien durch Experten leicht zu beeinflussen, setzt Schicktanz entgegen, dass auch Laien in der Lage seien, sich relativ schnell in komplexe medizinethische Sachverhalte einzuarbeiten.

Eine Politikberatung nur durch Experten setzte sich dagegen dem Einwand der Technokratie aus. Zwar sind Laiengruppen nicht so effizient wie Expertengremien, dieser Schwäche könne aber auch etwa durch eine stärkere Institutionalisierung entgegengewirkt werden. Einschränkend bemerkt Schicktanz, dass kleinere Laiengruppen aufgrund ihrer begrenzten Repräsentativität als Beratungsform komplementär zu den Expertengremien zu denken sind und diese nicht ersetzen können.

\section{Fazit}

Resümierend kann festgehalten werden, dass ethische Politikberatung immer beides umfasst, eine wissensbasierte Ebene, die die Erkenntnisse der Wissenschaften fruchtbar macht, und eine bewertende Ebene, die in Abhängigkeit von religiösen, weltanschaulichen und kulturellen Hintergründen formuliert wird. Diese Werte-Ebene ist zentral, auch und gerade in einem liberalen Verfassungsstaat.

Eine Negierung von Werten, insbesondere der Menschenwürde und Grundfreiheiten, würde zu Intoleranz und Unfreiheit führen. Selbst die eigene Bindung kann im Sinne einer übergeordneten Maximierung menschenwürdiger und freiheitlicher Lebensbedingungen vonnöten sein. Welchen Rat die Politik am Ende befolgt, ist den demokratischen Institutionen, insbesondere dem Parlament, vorbehalten.

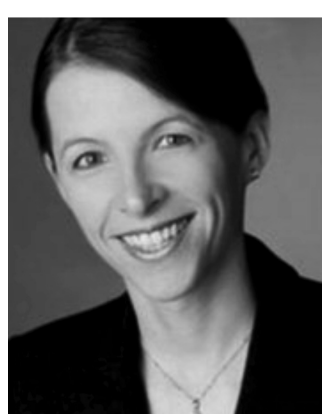

Dr. iur. A. Katarina Weilert, LL.M. ist wissenschaftliche Referentin an der Forschungsstätte der Evangelischen Studiengemeinschaft e.V. (FEST) in Heidelberg, Institut für interdisziplinäre Forschung. E-Mail: katarina. weilert@fest-heidelberg.de. 